KYUNGPOOK Math. J. 52(2012), 189-200

http://dx.doi.org/10.5666/KMJ.2012.52.2.189

\title{
On I-Convergent Double Sequences of Fuzzy Real Numbers
}

\author{
Binod Chandra Tripathy And Bipul SARMA* \\ Mathematical Sciences Division, Institute of Advanced Study in Science and Tech- \\ nology, Paschim Boragaon, Garchuk, Guwahati-781035, India \\ e-mail : tripathybc@yahoo.com, tripathybc@rediffmail.com \\ and sarmabipul010yahoo.co.in
}

Abstract. In this article we introduce the class of $I$-convergent double sequences of fuzzy real numbers. We have studied different properties like solidness, symmetricity, monotone, sequence algebra etc. We prove that the class of $I$-convergent double sequences of fuzzy real numbers is a complete metric spaces.

\section{Introduction}

The concept of fuzzy sets was first introduced by L. A. Zadeh in the year 1965 . It has a wide range of applications in almost all the branches of science, where mathematics is used. The notion of fuzzyness are used by the researchers in Cybernetics, Artficail Intelligence, Expert Systems and fuzzy control, Pattern recognition, Operations research, Decision making, Image analysis, Projectiles, Probability theory, Agriculture, Weather forecasting etc. The fuzzy analogue of all the subjects of mathematical science has been investigated. It attracted many workers on sequence spaces and summability theory to introduce different types of fuzzy sequence spaces and study their different properties. Our studies are based on the linear spaces of sequences of fuzzy real numbers which are very important for the higher level studies in Quantum mechanics, Partical physics, Statistical mechanics etc. Different classes of sequences of fuzzy numbers have been investigated by Altin, Et and Basarir [1], Altin, Mursaleen and Altinok [2], Altinok, Colak and Et [3], Colak, Altinok and Et [6], Tripathy and Baruah ([15], [16]), Tripathy and Borgohain [17], Tripathy and Dutta ([19], [20]) and many others. The present work is motivated by the works on $I$-convergent sequences by Kostyrko, Salát and Wilczyñski [8], Tripathy and Hazarika ([22], [23], [24]), Tripathy and Mahanta [25], Tripathy and Tripathy [28] and others.

* Corresponding Author.

Received September 25, 2010; accepted November 16, 2010.

2010 Mathematics Subject Classification: 40A05, 40A35 , 40D25, 46A45.

Key words and phrases: I-convergence, solid space, symmetric space, sequence algebra, completeness.

The work of the authors was financially supported by Council of Scientific \& Industrial Research, India, vide project No. 25(0139)/05/EMR-II. 
Throughout, a double sequence is denoted by $A=\left\langle X_{n k}\right\rangle$, a double infinite array of elements $X_{n k}$, where each $X_{n k}$ is a fuzzy real number.

The notion of sequence spaces has been investigated from different aspects by Tripathy, Choudhary and Sarma [18], Tripathy and Dutta [21] and others in the recent years. The initial works on double sequences is found in Bromwich [5]. Later on it was studied by Basarir and Sonalcan [4], Hardy [7], Moricz [9], Moricz and Rhoades [10], Tripathy [13], Tripathy and Dutta ([19], [20]) Tripathy and Tripathy [28] and many others. Hardy [7] introduced the notion of regular convergence for double sequences.

The notion of $I$-convergence of real valued sequence was studied at the initial

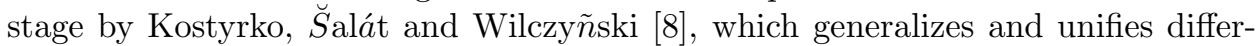
ent notions of convergence of sequences. The notion of $I$-convergence of double sequences was introduced by Tripathy and Tripathy [28].

\section{Definitions and background}

Definition 2.1. Let $X$ be a non-empty set, then a non-void class $I \subseteq 2^{X}$ (power set of $X$ ) is called an ideal if $I$ is additive (i.e. $A, B \in I \Rightarrow A \cup B \in I$ ) and hereditary (i.e. $A \in I$ and $B \subseteq A \Rightarrow B \in I$ ).

Definition 2.2. An ideal $I \subseteq 2^{X}$ is said to be non-trivial if $I \neq 2^{X}$. A non-trivial ideal $I$ is said to be admissible if $I$ contains every finite subset of $N$. A non trivial ideal $I$ is said to be maximal if there does not exist any non trivial ideal $J \neq I$ containing $I$ as a subset.

Definition 2.3. Let $X$ be a non-empty set, then a non-void class $\mathcal{F} \subseteq 2^{X}$ is said to be a filter in $X$ if $\emptyset \notin \mathcal{F} ; A, B \in \mathcal{F} \Rightarrow A \cap B \in \mathcal{F}$ and $A \in \mathcal{F}, A \subseteq B \Rightarrow B \in \mathcal{F}$.

For any ideal $I$, there is a filter $\mathcal{F}(I)$ corresponding to $I$, given by

$$
\mathcal{F}(I)=\{K \subseteq N: N \backslash K \in I\} .
$$

Example 2.1. (a) Let $I=I_{f}$, class of all finite subsets of $N$. Then $I_{f}$ is a nontrivial admissible ideal of $2^{N}$.

(b) Let $A \subset N$. If $\delta(A)=\lim _{n \rightarrow \infty} \frac{1}{n} \sum_{k=1}^{n} \chi_{A}(k)$ exists, then the class $I_{\delta}$ of all $A \subset N$ with $\delta(A)=0$ forms a non-trivial admissible ideal of $2^{N}$.

This is known as asymptotic density of $A$ and the type of convergence related to this notion is known as statistical convergence. Different classes of statistically convergent sequence spaces have been investigated and related with characterization of matrix classes by Rath and Tripathy [11], Tripathy ([12], [13], [14]), Tripathy and Sen ([26], [27]) and many others.

(c) Let $A \subset N$ and $s_{n}=\sum_{k=1}^{n} \frac{1}{k}$, for all $n \in N$. If $d(A)=\lim _{n \rightarrow \infty} \frac{1}{s_{n}} \sum_{k=1}^{n} \frac{\chi_{A}(k)}{k}$ exists, then the class $I_{d}$ of all $A \subset N$ with $d(A)=0$ forms a non-trivial admissible ideal. 
Definition 2.4. A subset $E$ of $N \times N$ is said to have density $\rho(E)$, if

$$
\rho(E)=\lim _{p, q \rightarrow \infty} \sum_{n=1}^{p} \sum_{k=1}^{q} \chi_{E}(n, k) \text { exists }
$$

where $\chi_{E}$ is the characteristic function of $E$. This is known as the asymptotic density of $E$.

Definition 2.5. Let $p, q \geq 0$ and $s, t \geq 1$, be integers. Let $D \subseteq N \times N$ and $D(p+1, p+t ; q+1, q+s)=$ card $\{(n, k) \in D: p+1 \leq n \leq p+t$ and $q+1 \leq k \leq q+s\}$. Put $\beta_{t, s}=\liminf _{p, q \rightarrow \infty} D(p+1, p+t ; q+1, q+s)$ and $\beta^{t, s}=\limsup _{p, q \rightarrow \infty} D(p+1, p+t ; q+1, q+s)$. Let $\underline{u}(D)=\lim _{t, s \rightarrow \infty} \frac{\beta_{t, s}}{t s}$ exists and $\bar{u}(D)=\lim _{t, s \rightarrow \infty} \frac{\beta^{t, s} t s}{t s}$ exists. If $\underline{u}(D)=\bar{u}(D)=u(D)$, say. Then $u(D)$ is called the uniform density of $D$.

Definition 2.6. Let $s_{n}=\sum_{k=1}^{n} \frac{1}{k}$. Then a subset $E$ of $N \times N$ is said to have logarithmic density $\rho^{*}(E)$ if

$$
\rho^{*}(E)=\lim _{p, q \rightarrow \infty} \frac{1}{s_{p} s_{q}} \sum_{n=1}^{p} \sum_{k=1}^{q} \frac{\chi_{E}(n, k)}{n k} \text { exists. }
$$

Since $s_{n}=\sum_{k=1}^{n} \frac{1}{k}=\log n+\gamma+\bigcirc\left(\frac{1}{n}\right)$, where $\gamma$ is the Euler's constant, the above expression is equivalent to the following:

$$
\rho^{*}(E)=\lim _{p, q \rightarrow \infty} \frac{1}{\log p} \frac{1}{\log q} \sum_{n=1}^{p} \sum_{k=1}^{q} \frac{\chi_{E}(n, k)}{n k} \text { exists. }
$$

Let $D$ denote the set of all closed and bounded intervals $X=\left[a_{1}, a_{2}\right]$ on the real line $R$. For $X=\left[a_{1}, a_{2}\right] \in D$ and $Y=\left[b_{1}, b_{2}\right] \in D$, define $d(X, Y)$ by

$$
d(X, Y)=\max \left(\left|a_{1}-b_{1}\right|,\left|a_{2}-b_{2}\right|\right) .
$$

It is known that $(D, d)$ is a complete metric space.

Definition 2.7. A fuzzy real number $X$ is a fuzzy set on $R$, i.e. a mapping $X: R \rightarrow L(=[0,1])$ associating each real number $t$ with its grade of membership $X(t)$.

Definition 2.8. The $\alpha$-level set of a fuzzy real number $X, 0<\alpha \leq 1$ denoted by $X^{\alpha}$ is defined as $X^{\alpha}=\{t \in R: X(t) \geq \alpha\}$. The 0-level set is the closure of the strong 0-cut, i.e. 0-level set $=c l\{t \in R: X(t)>0\}$.

Definition 2.9. A fuzzy real number $X$ is called convex, if $X(t) \geq X(s) \wedge X(r)=$ $\min (X(s), X(r))$, where $s<t<r$. If there exists $t_{0} \in R$ such that $X\left(t_{0}\right)=1$, then the fuzzy real number $X$ is called normal. A fuzzy real number $X$ is said to be 
upper semi-continuous if for each $\varepsilon>0, X^{-1}([0, a+\varepsilon))$, for all $a \in L$ is open in the usual topology of $R$. The set of all upper semi-continuous, normal, convex fuzzy number is denoted by $L(R)$.

Definition 2.10. The absolute value $|X|$ of $X \in L(R)$ is defined by

$$
|X|(t)= \begin{cases}\max \{X(t), X(-t)\}, & \text { if } t \geq 0 \\ 0, & \text { if } t<0\end{cases}
$$

Let $\bar{d}: L(R) \times L(R) \rightarrow R$ be defined by

$$
\bar{d}(X, Y)=\sup _{0 \leq \alpha \leq 1} d\left(X^{\alpha}, Y^{\alpha}\right) .
$$

Then $\bar{d}$ defines a metric on $L(R)$.

The additive identity and multiplicative identity in $L(R)$ are denoted by $\overline{0}$ and $\overline{1}$ respectively.

Definition 2.11. A sequence $\left(X_{n}\right)$ of fuzzy real numbers is said to be convergent to the fuzzy real number $X_{0}$, if for every $\varepsilon>0$, there exists $n_{0} \in N$ such that $\bar{d}\left(X_{n}, X_{0}\right)<\varepsilon$ for all $n \geq n_{0}$.

Definition 2.12. A fuzzy real-valued sequence space $E^{F}$ is said to be solid if $\left(Y_{n}\right) \in E^{F}$ whenever $\left(X_{n}\right) \in E^{F}$ and $\left|Y_{n}\right| \leq\left|X_{n}\right|$, for all $n \in N$.

Definition 2.13. Let $K=\left\{\left(n_{i}, k_{i}\right): i \in N ; n_{1}<n_{2}<n_{3}<\ldots\right.$ and $\left.k_{1}<k_{2}<k_{3}<\ldots\right\} \subseteq N \times N$ and $E^{F}$ be a double sequence space. A $K$-step space of $E^{F}$ is a sequence space $\lambda_{K}^{E}=\left\{\left\langle X_{n_{i} k_{i}}\right\rangle \in{ }_{2} w^{F}:<X_{n k}>\in E^{F}\right\}$.

Definition 2.14. A canonical pre-image of a sequence $<X_{n_{i} k_{i}}>\in E^{F}$ is a sequence $<Y_{n k}>$ defined as follows:

$$
Y_{n k}= \begin{cases}X_{n k}, & \text { if }(n, k) \in K, \\ 0, \text { otherwise. } & \end{cases}
$$

A canonical pre-image of a step space $\lambda_{K}^{E}$ is a set of canonical pre-images of all elements in $\lambda_{K}^{E}$.

Definition 2.15. A sequence space $E^{F}$ is said to be monotone if it contains the canonical pre-images of all its step spaces.

Definition 2.16. A double sequence space $E^{F}$ is said to be symmetric if $<X_{n, k}>\in E$ implies $\left\langle X_{\pi(n, k)}>\in E\right.$, where $\pi$ is a permutation of $N \times N$.

Definition 2.17. A sequence $X=\left(X_{n}\right)$ of fuzzy numbers is said to be $I$ convergent if there exists a fuzzy number $X_{0}$ such that for all $\varepsilon>0$, the set 
$\left\{n \in N: \bar{d}\left(X_{n}, X_{0}\right) \geq \varepsilon\right\} \in I$. We write $I-\lim X_{n}=X_{0}$.

Definition 2.18. A sequence $\left(X_{n}\right)$ of fuzzy numbers is said to be $I^{*}$-convergent to $X_{0}\left(I^{*}-\lim X_{n}=X_{0}\right)$ if there is a set $\left\{n_{1}<n_{2}<\ldots \ldots\right\} \in \mathcal{F}(I)$ such that $\lim _{i \rightarrow \infty} X_{n_{i}}=X_{0}$.

Definition 2.19. A sequence $\left(X_{n}\right)$ of fuzzy numbers is said to be $I$-bounded if there exists a real number $\mu$ such that the set $\left\{n \in N: \bar{d}\left(X_{n}, \overline{0}\right)>\mu\right\} \in I$.

If $I=I_{f}$, then $I_{f}$ convergence coincides with the usual convergence of sequences of fuzzy numbers. If $I=I_{d}\left(I_{\delta}\right)$, then $I_{d}\left(I_{\delta}\right)$ convergence coincides with statistical convergence (logarithmic convergence) of sequences of fuzzy numbers. If $I=I_{u}, I_{u}$ convergence is said to be uniform convergence of sequences of fuzzy numbers.

Let $c_{F}^{I},\left(c_{0}^{I}\right)_{F}$ and $\left(\ell_{\infty}^{I}\right)_{F}$ denote the classes of fuzzy real-valued $I$-convergent, $I$-null and $I$-bounded sequences respectively. proper.

It is clear from the definitions that $\left(c_{0}^{I}\right)_{F} \subset c_{F}^{I} \subset\left(\ell_{\infty}^{I}\right)_{F}$ and the inclusions are

It can be easily shown that $\left(\ell_{\infty}\right)_{F}$ is complete with respect to the metric $\rho$ defined by

$$
\rho(X, Y)=\sup _{n} \bar{d}\left(X_{n}, Y_{n}\right), \text { where } X=\left(X_{n}\right), Y=\left(Y_{n}\right) \in\left(\ell_{\infty}\right)_{F} .
$$

Remark 2.1. A sequence space $E^{F}$ is solid implies $E^{F}$ is monotone.

Lemma 2.1. If $I \subset 2^{N}$ is a maximal ideal, then for each $A \subset N$ we have either $A \in I$ or $N \backslash A \in I$. (see for instance lemma 5.1 [8]).

\section{I-convergent double sequences of fuzzy numbers}

The notion of $I$-convergence of double sequences was introduced by Tripathy and Tripathy [28]. In this section we introduce some definition of $I$-convergence of sequences of fuzzy numbers. In order to distinguish between the ideals of $2^{N}$ and $2^{N \times N}$, we shall denote the ideals of $2^{N}$ by $I$ and that of $2^{N \times N}$ by $I_{2}$.

Definition 3.1. Let $I_{2}$ be an ideal of $2^{N \times N}$. A double sequence $<X_{n k}>$ of fuzzy numbers is said to be $I$-convergent to $X_{0}$ in Pringsheim's sense if for every $\varepsilon>0$,

$$
\left\{(n, k) \in N \times N: \bar{d}\left(X_{n k}, X_{0}\right) \geq \varepsilon\right\} \in I_{2} .
$$

For $X_{0}=\overline{0}$, it is called $I$-null in Pringsheim's sense.

Definition 3.2. Let $I_{2}$ be an ideal of $2^{N \times N}$ and $I$ be an ideal of $2^{N}$. A double sequence $\left\langle X_{n k}\right\rangle$ of fuzzy real numbers is said to be regularly $I$-convergent to a number $X_{0}$ if it is I-convergent in Pringsheim's sense to $X_{0}$ and for every $\varepsilon>0$, the followings hold.

For each $k \in N$, there exists $L_{k} \in L(R)$ such that $\left\{n \in N: \bar{d}\left(X_{n k}, L_{k}\right) \geq \varepsilon\right\} \in I$, and for each $n \in N$, there exists $M_{n} \in L(R)$ such that $\left\{k \in N: \bar{d}\left(X_{n k}, M_{n}\right) \geq \varepsilon\right\} \in$ 
$I$.

When $X_{0}=L_{k}=L_{n}=\overline{0}$, for all $n, k \in N$ the sequence $\left\langle X_{n k}>\right.$ is said to be regularly $I$-null.

Definition 3.3. A double sequence $\left\langle X_{n k}>\right.$ of fuzzy real numbers is said to be $I$-Cauchy if for every $\varepsilon>0$, there exist $s=s(\varepsilon), t=t(\varepsilon) \in N$ such that $\left\{(n, k) \in N \times N: \bar{d}\left(X_{n k}, X_{s t}\right) \geq \varepsilon\right\} \in I_{2}$.

Definition 3.4. A double sequence $\left\langle X_{n k}>\right.$ of fuzzy real numbers is said to be $I$ bounded if there exists a real number $K>0$ such that $\left\{(n, k) \in N \times N: \bar{d}\left(X_{n k}, \overline{0}\right) \geq\right.$ $K\} \in I_{2}$.

Throughout the article $\left({ }_{2} \ell_{\infty}^{I}\right)_{F},{ }_{2} c_{F}^{I},\left({ }_{2} c_{0}^{I}\right)_{F},\left({ }_{2} c^{I}\right)_{F}^{R}$ and $\left({ }_{2} c_{0}^{I}\right)_{F}^{R}$ will denote the classes of $I$-bounded, $I$-convergent in Pringsheim's sense, $I$-null in Pringsheim's sense, regularly $I$-convergent and regularly $I$-null double sequences of fuzzy numbers respectively.

$$
\begin{aligned}
\text { Also we define } & \left({ }_{2} c^{I}\right)_{F}^{B}={ }_{2} c_{F}^{I} \cap\left({ }_{2} \ell_{\infty}\right)_{F} ; \quad\left({ }_{2} c_{0}^{I}\right)_{F}^{B}=\left({ }_{2} c_{0}^{I}\right)_{F} \cap\left({ }_{2} \ell_{\infty}\right)_{F} \\
& \left.\left({ }_{2} c^{I}\right)_{F}^{B R}={ }_{2} c^{I}\right)_{F}^{R} \cap\left({ }_{2} \ell_{\infty}\right)_{F} ; \quad\left({ }_{2} c_{0}^{I}\right)_{F}^{B R}=\left({ }_{2} c_{0}^{I}\right)_{F}^{R} \cap\left({ }_{2} \ell_{\infty}\right)_{F} .
\end{aligned}
$$

Definition 3.5. Let $X=<X_{n k}>$ and $Y=<Y_{n k}>$ be two double sequences. Then we say that $X_{n k}=Y_{n k}$ for almost all $n$ and $k$ relative to $I_{2}$ (in short a.a.n \& $\left.k r . I_{2}\right)$ if

$$
\left\{(n, k) \in N \times N: X_{n k} \neq Y_{n k}\right\} \in I_{2} .
$$

\section{Main results}

Theorem 4.1. The classes of sequences $\left({ }_{2} c^{I}\right)_{F}^{B},\left({ }_{2} c^{I}\right)_{F}^{B R},\left({ }_{2} c_{0}^{I}\right)_{F}^{B},\left({ }_{2} c_{0}^{I}\right)_{F}^{B R}$ are complete metric spaces with respect to the metric

$$
\rho(X, Y)=\sup _{n, k} \bar{d}\left(X_{n k}, Y_{n k}\right),
$$

where $X=<X_{n k}>, Y=<Y_{n k}>$.

Proof. We prove the result for the class of sequences $\left({ }_{2} c^{I}\right)_{F}^{B}$. Let $\left(X^{i}\right)$ be a Cauchy sequence in $\left({ }_{2} c^{I}\right)_{F}^{B}$. Then $X^{i} \rightarrow X$ in $\left({ }_{2} \ell_{\infty}\right)_{F}$. Let $I-\lim X_{n k}^{i}=L_{i}$ for each $i \in N$. We are to show that

(i) $\left(L_{i}\right)$ is convergent, say to $L$.

(ii) $I-\lim X_{n k}=L$.

Since $\left(X^{i}\right)$ is Cauchy, so for each $\varepsilon>0$, there exists $n_{0} \in N$ such that

$$
\rho\left(X^{i}, X^{j}\right)<\frac{\varepsilon}{3} \text { for all } i, j \geq n_{0} .
$$

Now there exist sets $E_{i}$ and $E_{j}$ in $I_{2}$ such that

$$
E_{i}=\left\{(n, k): \bar{d}\left(X_{n k}^{i}, L_{i}\right) \geq \frac{\varepsilon}{3}\right\}
$$




$$
E_{j}=\left\{(n, k): \bar{d}\left(X_{n k}^{j}, L_{j}\right) \geq \frac{\varepsilon}{3}\right\} .
$$

Consider $i, j \geq n_{0}$ and $(n, k) \notin E_{i} \cap E_{j}$. Then

$$
\begin{aligned}
\bar{d}\left(L_{i}, L_{j}\right) & \leq \bar{d}\left(X_{n k}^{i}, L_{i}\right)+\bar{d}\left(X_{n k}^{i}, X_{n k}^{j}\right)+\bar{d}\left(X_{n k}^{j}, L_{j}\right) \\
& <\varepsilon
\end{aligned}
$$

Thus $\left(L_{i}\right)$ is a Cauchy sequence of fuzzy real numbers, so it is convergent.

Let $\lim _{j \rightarrow \infty} L_{j}=L$. Let $\eta>0$ be given, then we can find $m_{0}$ such that

$$
\bar{d}\left(L_{j}, L\right)<\frac{\eta}{3} \text { for all } j>m_{0} .
$$

Also $X^{i} \rightarrow X$ as $i \rightarrow \infty$. Thus $\rho\left(X^{i}, X\right)<\frac{\eta}{3}$ for all $i>m_{0}$.

Since $<X_{n k}^{j}>$ is $I$-convergent to $L_{j}$ so there exists $D \in I_{2}$ such that for each $(n, k) \notin D$,

$$
\bar{d}\left(X_{n k}^{i}, L_{j}\right)<\frac{\eta}{3} .
$$

Without loss of generality let $j>m_{0}$. Then for all $(n, k) \notin D$,

$$
\bar{d}\left(X_{n k}, L\right) \leq \bar{d}\left(X_{n k}, X_{n k}^{j}\right)+\bar{d}\left(X_{n k}^{j}, L_{j}\right)+\bar{d}\left(L_{j}, L\right)<\eta .
$$

Hence $<X_{n k}>$ is $I$-convergent to $L$.

Theorem 4.2. The classes of sequences $\left({ }_{2} c_{0}^{I}\right)_{F},\left({ }_{2} c_{0}^{I}\right)_{F}^{B},\left({ }_{2} c_{0}^{I}\right)_{F}^{R},\left({ }_{2} c_{0}^{I}\right)_{F}^{B R}$ and $\left({ }_{2} \ell_{\infty}^{I}\right)_{F}$ are solid and hence monotone.

Proof. We prove the result for the class of sequences $\left({ }_{2} \ell_{\infty}^{I}\right)_{F}$. For the other classes of sequences the proof will follow similarly. Let $\left\langle X_{n k}>\in\left({ }_{2} \ell_{\infty}^{I}\right)_{F}\right.$ and $\left\langle Y_{n k}\right\rangle$ be such that $\left|Y_{n k}\right| \leq\left|X_{n k}\right|$ for all $n, k \in N$. Then for a given $\mu>0, A=\{(n, k) \in$ $\left.N \times N: \bar{d}\left(X_{n k}, \overline{0}\right) \geq \mu\right\} \in I_{2}$.

Now $B=\left\{(n, k) \in N \times N: \bar{d}\left(Y_{n k}, \overline{0}\right) \geq \mu\right\} \subseteq A$. Thus $B \in I_{2}$ and so $<Y_{n k}>\in\left({ }_{2} \ell_{\infty}^{I}\right)_{F}$. The class of sequences $\left.{ }_{2} \ell_{\infty}^{I}\right)_{F}$ is monotone follows by Remark 2.1 .

Property 4.3. Let I be not maximal, then the classes of sequences ${ }_{2} c_{F}^{I},\left({ }_{2} c^{I}\right)_{F}^{B}$, $\left({ }_{2} c^{I}\right)_{F}^{R}$ and $\left({ }_{2} c^{I}\right)_{F}^{B R}$ are not monotone and hence not solid.

To show the above property consider the following example.

Example 4.1. Consider the space ${ }_{2} c_{F}^{I}$. Let $I_{2}$ be the ideal of those subsets $E$ of $N \times N$ such that

$$
\rho^{*}(E)=\lim _{p, q \rightarrow \infty} \frac{1}{s_{p} s_{q}} \sum_{n=1}^{p} \sum_{k=1}^{q} \chi_{E}(p, q)=0 .
$$


For all $n, k \in N$ define the fuzzy sequence $<X_{n k}>$ by

$$
X_{n k}(t)= \begin{cases}t, & \text { for } 0 \leq t \leq 1 \\ -t+2, & \text { for } 1 \leq t \leq 2 \\ 0, & \text { otherwise }\end{cases}
$$

Then the sequence $\left\langle X_{n k}\right\rangle$ is logarithmically convergent. Let $J$ be a subset of $N \times N$ such that $J=\{(n, k): n>k\}$.

Let the sequence $<Y_{n k}>$ be defined by

$$
Y_{n k}=\left\{\begin{array}{l}
X_{n k}, \text { if }(n, k) \in J \\
\overline{0}, \text { otherwise }
\end{array}\right.
$$

Then $\left\langle Y_{n k}\right\rangle$ belongs to the canonical pre-image of $J$-step space of $\left({ }_{2} c^{I}\right)_{F}$, but $<Y_{n k}>\notin\left({ }_{2} c^{I}\right)_{F}$. Thus $\left.{ }_{2} c^{I}\right)_{F}$ is not monotone and hence not solid.

Similarly it can be shown that the other classes of sequences are not monotone and hence are not solid.

Property 4.4. If $I_{2}$ is neither maximal nor $I_{2}=\left(I_{2}\right)_{f}$ (ideal of finite subsets of $N \times N)$, then ${ }_{2} c_{F}^{I},\left({ }_{2} c^{I}\right)_{F}^{B},\left({ }_{2} c^{I}\right)_{F}^{R},\left({ }_{2} c^{I}\right)_{F}^{B R},\left({ }_{2} c_{0}^{I}\right)_{F},\left({ }_{2} c_{0}^{I}\right)_{F}^{B},\left({ }_{2} c_{0}^{I}\right)_{F}^{R}$ and $\left({ }_{2} c_{0}^{I}\right)_{F}^{B R}$ are not symmetric.

To prove the result consider the following example.

Example 4.2. Consider the classes of sequences $\left({ }_{2} c^{I}\right)_{F}$. Let $I_{2}$ be the ideal of those subsets $E$ of $N \times N$ such that

$$
\rho(E)=\lim _{p, q \rightarrow \infty} \frac{1}{p q} \sum_{n=1}^{p} \sum_{k=1}^{q} \chi_{E}(p, q)=0 .
$$

Consider the fuzzy sequence $<X_{n k}>$ defined by

$$
X_{1 k}=\overline{0}
$$

and

$$
X_{n k}(t)= \begin{cases}t-1, & \text { for } 1 \leq t \leq 2 \\ -t+3, & \text { for } 2 \leq t \leq 3 \\ 0, & \text { otherwise }\end{cases}
$$

for other values of $n \in N$. Then $<X_{n k}>\in\left({ }_{2} c^{I}\right)_{F}$.

Let $\left.<Y_{n k}\right\rangle$ be a rearrangement of $\left\langle X_{n k}\right\rangle$ defined as follows:

For $n$ even and all $k \in N$,

$$
Y_{n k}(t)= \begin{cases}t-1, & \text { for } 1 \leq t \leq 2 \\ -t+3, & \text { for } 2 \leq t \leq 3 \\ 0, & \text { otherwise }\end{cases}
$$


and $Y_{n k}=\overline{0}$, otherwise.

Then $\left\langle Y_{n k}>\notin\left({ }_{2} c^{I}\right)_{F}\right.$. Hence the space $\left({ }_{2} c^{I}\right)_{F}$ is not symmetric.

Similarly one can construct examples for the other classes of sequences.

Property 4.5. If $I_{2}$ is neither maximal nor $I_{2}=\left(I_{2}\right)_{f}$, then $\left({ }_{2} \ell_{\infty}^{I}\right)_{F}$ is not symmetric.

Proof. To establish the result consider the following example.

Example 4.3. Let $I_{2}$ be the ideal of those subsets $E$ of $N \times N$ such that

$$
\rho(E)=\lim _{p, q \rightarrow \infty} \frac{1}{p q} \sum_{n=1}^{p} \sum_{k=1}^{q} \chi_{E}(p, q)=0 .
$$

Let the sequences of fuzzy numbers $\left\langle X_{n k}>\right.$ be defined by For $n$ even and $k=i^{2}, i \in N$,

$$
X_{n k}(t)= \begin{cases}t, & \text { for } 0 \leq t \leq 1 \\ -t i^{-1}+1+i^{-1}, & \text { for } 1 \leq t \leq n \\ 0, & \text { otherwise }\end{cases}
$$

and $X_{n k}=\overline{0}$, otherwise.

Then $<X_{n k}>\in\left({ }_{2} \ell_{\infty}^{I}\right)_{F}$.

Let $\left\langle Y_{n k}\right\rangle$ be a rearrangement of $\left\langle X_{n k}\right\rangle$ defined as follows:

For $k$ odd and all $n \in N$,

$$
Y_{n k}(t)= \begin{cases}t, & \text { for } 0 \leq t \leq 1 \\ -2 t(k+1)^{-1}+1+2(k+1)^{-1}, & \text { for } 1 \leq t \leq n \\ 0, & \text { otherwise }\end{cases}
$$

Then $<Y_{n k}>\notin\left({ }_{2} \ell_{\infty}^{I}\right)_{F}$. Hence $\left({ }_{2} \ell_{\infty}^{I}\right)_{F}$ is not symmetric.

Theorem 4.6. The classes of sequences ${ }_{2} c_{F}^{I},\left({ }_{2} c^{I}\right)_{F}^{B},\left({ }_{2} c^{I}\right)_{F}^{R},\left({ }_{2} c^{I}\right)_{F}^{B R},\left({ }_{2} c_{0}^{I}\right)_{F}$, $\left({ }_{2} c_{0}^{I}\right)_{F}^{B},\left({ }_{2} c_{0}^{I}\right)_{F}^{R}$ and $\left({ }_{2} c_{0}^{I}\right)_{F}^{B R}$ are sequence algebra.

Proof. Consider the classes of sequences $\left({ }_{2} c_{0}^{I}\right)_{F}$. Let $\left\langle X_{n k}\right\rangle,\left\langle Y_{n k}\right\rangle \in\left({ }_{2} c_{0}^{I}\right)_{F}$. Then corresponding to the ideal $I_{2}$ there exist a filter $\mathcal{F}(I)$ such that

$$
\begin{aligned}
& A=\left\{(n, k): \bar{d}\left(X_{n k}, \overline{0}\right)<\varepsilon\right\} \in \mathcal{F}\left(I_{2}\right), \\
& B=\left\{(n, k): \bar{d}\left(Y_{n k}, \overline{0}\right)<\varepsilon\right\} \in \mathcal{F}\left(I_{2}\right) .
\end{aligned}
$$

Let $\left[X_{n k}\right]^{\alpha}=[a, b], \quad\left[Y_{n k}\right]^{\alpha}=[c, d]$, then

$$
\left[X_{n k} Y_{n k}\right]^{\alpha}=[\min (a c, a d, b c, b d), \max (a c, a d, b c, b d)]=[a d, b c](\text { suppose }) .
$$


Now $\bar{d}\left(X_{n k} Y_{n k}, \overline{0}\right)=\max \{|a d|,|b c|\}, \bar{d}\left(X_{n k}, \overline{0}\right)=\max \{|a|,|b|\}, \bar{d}\left(Y_{n k}, \overline{0}\right)=$ $\max \{|c|,|d|\}$.

Now for all values of $\bar{d}\left(X_{n k} Y_{n k}, \overline{0}\right), \bar{d}\left(X_{n k}, \overline{0}\right), \bar{d}\left(Y_{n k}, \overline{0}\right)$ we have

$$
\bar{d}\left(X_{n k} Y_{n k}, \overline{0}\right) \leq \bar{d}\left(X_{n k}, \overline{0}\right) \bar{d}\left(Y_{n k}, \overline{0}\right)
$$

Hence for all $(n, k) \in A \cap B$,

$$
\begin{aligned}
\bar{d}\left(X_{n k} Y_{n k}, \overline{0}\right) & \leq \bar{d}\left(X_{n k}, \overline{0}\right) \bar{d}\left(Y_{n k}, \overline{0}\right) \\
& <\varepsilon^{2} .
\end{aligned}
$$

Since $A \cap B \in \mathcal{F}\left(I_{2}\right)$ so $<X_{n k} Y_{n k}>\in\left({ }_{2} c_{0}^{I}\right)_{F}$.

Similarly it can be shown that the other classes of sequences are also sequence algebra.

Property 4.7. If $I$ is not maximal then the classes of sequences ${ }_{2} c_{F}^{I},\left({ }_{2} c^{I}\right)_{F}^{B}$, $\left({ }_{2} c^{I}\right)_{F}^{R},\left({ }_{2} c^{I}\right)_{F}^{B R},\left({ }_{2} c_{0}^{I}\right)_{F},\left({ }_{2} c_{0}^{I}\right)_{F}^{B},\left({ }_{2} c_{0}^{I}\right)_{F}^{R}$ and $\left({ }_{2} c_{0}^{I}\right)_{F}^{B R}$ are not convergence free.

Proof. The result follows from the following example.

Example 4.4. Consider the classes of sequences ${ }_{2} c_{F}^{I}$. Let $I_{2}$ be the ideal of finite subsets of $N \times N$. Let us consider the sequence $\left\langle X_{n k}\right\rangle$ defined by

$X_{1 k}=\overline{0}$ for all $k \in N$ and for $n \neq 1$ and all $k \in N$,

$$
X_{n k}(t)= \begin{cases}t+1, & \text { for }-1 \leq t \leq 0, \\ -n(n+1)^{-1} t+1, & \text { for } 0 \leq t \leq 1+n^{-1}, \\ 0, & \text { otherwise }\end{cases}
$$

Let the sequence $\left\langle Y_{n k}>\right.$ be defined by

$Y_{1 k}=\overline{0}$ for all $k \in N$ and for $n \neq 1$ and all $k \in N$,

$$
Y_{n k}(t)= \begin{cases}1, & \text { for } 0 \leq t \leq 1 \\ (n-t)(n-1)^{-1}, & \text { for } 1 \leq t \leq n, \\ 0, & \text { otherwise. }\end{cases}
$$

Here $\left\langle X_{n k}>\in{ }_{2} c_{F}^{I}\right.$ but $\left\langle Y_{n k}>\notin{ }_{2} c_{F}^{I}\right.$. Hence the space ${ }_{2} c_{F}^{I}$ is not convergence free.

Similarly it can be shown that the other spaces are not convergence free.

\section{Conclusion}

In this paper we have introduced and studied the notion of $I$-convergent double sequences of fuzzy real numbers. We have established the completeness property of the introduced class of sequences. We have verified some algebraic and topological properties. The difference between sequences of crisp numbers and the classes of sequences of fuzzy numbers are given by providing suitable examples. The introduced notion can be applied for further investigations from different aspects. 


\section{References}

[1] Y. Altin, M. Et and M. Basarir, on some generalized difference sequences of fuzzy numbers, Kuwait J. Sci. Eng., 34(1A)(2007), 1-14.

[2] Y. Altin, M. Mursaleen and H. Altinok, Statistical summability $(C, 1)$ for sequences of fuzzy real numbers and a Tauberian theorem, Jour. Intell. Fuzzy Systems, 21(2010), 379-384.

[3] H. Altinok, R. Colak and M. Et, $\lambda$-Difference sequence spaces of fuzzy numbers, Fuzzy Sets Systems, 160(21)(2009), 3128-3139.

[4] M. Basarir and O. Sonalcan, On some double sequence spaces, J. Indian Acad. Math., 21(2)(1999), 193-200.

[5] T. J. I. Bromwich, An Introduction to the Theory of Infinite Series, MacMillan and Co. Ltd. New York, 1965.

[6] R. Colak, H. Altinok and M. Et, Generalized difference sequences of fuzzy numbers, Chaos Solitons Fractrals, 40(2009), 1106-1117.

[7] G. H. Hardy, On the convergence of certain multiple series, Proc. Camb. Phil. Soc., 19(1917), 86-95.

[8] P. Kostyrko, T. S̆alát and W. Wilczyñski, I-convergence, Real Anal. Exchange, 26(2000-2001), 669-686.

[9] F. Moricz, Extension of the spaces $c$ and $c_{0}$ from single to double sequences, Acta. Math. Hungerica, 57(1-2)(1991), 129-136.

[10] F. Moricz and B. E. Rhoades, Almost convergence of double sequences and strong regularity of summability matrices, Math. Proc. Camb. Phil. Soc., 104(1988), 283294.

[11] D. Rath and B. C. Tripathy, Matrix maps on sequence spaces associated with sets of integers, Indian J. Pure Appl. Math., 27(2)(1996), 197-206.

[12] B. C. Tripathy, Matrix transformations between some classes of sequences, Jour. Math. Anal. Appl., 206(3)(1997), 448-450.

[13] B. C. Tripathy, Statistically convergent double sequences, Tamkang J. Math., 34(3)(2003), 231-237.

[14] B. C. Tripathy, On generalized difference paranormed statistically convergent sequences, Indian J. Pure Appl. Math., 35(5)(2004), 655-663.

[15] B. C. Tripathy and A. Baruah, New type of difference sequence spaces of fuzzy real numbers, Math. Modell. Anal., 14(3)(2009), 391-397.

[16] B. C. Tripathy and A. Baruah, Lacunary statistically convergent and lacunary strongly convergent generalized difference sequences of fuzzy real numbers, Kyungpook Math. J., 50(2010), 565-574.

[17] B. C. Tripathy and S. Borgogain, The sequence space $m\left(M, \phi, \Delta_{m}^{n}, p\right)^{F}$, Math. Modell. Anal., 13(4)(2008), 577-586.

[18] B. C. Tripathy, B. Choudhary and B. Sarma, On some new type generalized difference sequence spaces, Kyungpook Math. J., 48(4)(2008), 613-622. 
[19] B. C. Tripathy and A. J. Dutta, On fuzzy real-valued double sequence spaces ${ }_{2} \ell_{F}^{p}$, Math. Comput. Modell., 46(9-10)(2007), 1294-1299.

[20] B. C. Tripathy and A. J. Dutta, Bounded variation double sequence space of fuzzy real numbers, Comput. Math. Appl., 59(2)(2010), 1031-1037.

[21] B. C. Tripathy and H. Dutta, On some new paranormed difference sequence spaces defined by Orlicz functions, Kyungpook Math. J., 50(2010), 59-69.

$[22]$ B. C. Tripathy and B. Hazarika, I-convergent sequence spaces associated with multiplier sequence spaces, Math. Ineq. Appl., 11(3)(2008), 543-548.

[23] B. C. Tripathy and B. Hazarika, Paranormed I-convergent sequences spaces, Math. Slovaca, 59(4)(2009), 485-494.

[24] B. C. Tripathy and B. Hazarika, I-convergent sequences spaces defined by Orlicz function, Acta Math. Appl. Sin. Eng. Ser., 27(1)(2011), 149-154.

[25] B. C. Tripathy and S. Mahanta, On I-acceleration convergence of sequences, Jour. Franklin Inst., 347(2010), 591-598.

[26] B. C. Tripathy and M. Sen, On generalized statistically convergent sequences, Indian J. Pure Appl. Math., 32(11)(2001), 1689-1694.

[27] B. C. Tripathy and M. Sen, Characterization of some matrix classes involving paranormed sequence spaces, Tamkang Jour. Math., 37(2)(2006), 155-162.

[28] B. K. Tripathy and B. C. Tripathy, On I-convergent double sequences, Soochow J. Math., 31(4)(2005), 549-560. 